\title{
Homologous series and parallel evolution problem
}

\author{
V. Suslov*, M. Ponomarenko, D. Rasskazov \\ Institute of Cytology and Genetics SB RAS, Novosibirsk, Russia \\ *e-mail:valya@bionet.nsc.ru
}

Key words: parallel evolution, transcription factor, Vavilov's law of homologous series

Motivation and Aim: The routine events of contradictions between the classic and molecular phylogenies of any taxa, irrespective of which reconstruction is closer to the truth, translocate the parallel evolution problem from the rare and facile event of convergence evolution associated with selection of similar environment to the homologous series phenomenon (HS), in which adaptation to a certain environment is only one of the reasons for the parallelism. HS arise due to the evolution of any trait in a limited space of possibilities (LSP). Various LSP allows to talk about the bouquet of the laws of HS. Current evolutionary synthesis incorporated only for those for whom the mechanisms of evolution of LSP were identified: Mueller-Haeckel and von Baer laws (explanation is the hourglass of ontogenesis) and HS for inherited traits and noninherited modification built by Lamarck and explained by genocoping according to Schmalhausen, Lukin or Turesson. Vavilov's law of HS remained outside synthesis because according to STE selection worked with traits, not HS. In 2016, we have shown that this is not so due to traits functional overlap of depending on which the HS must converge, diverge or stabilize even in a stable environment as autoadaptation [1]. Here we continued the search for the laws of HS evolution.

Methods: SNP_TATA_Comparator (http://beehive.bionet.nsc.ru) and the sample of TATA-boxes and composite elements (CE) with TATA-boxes used as [1].

Results: It is shown that clear HS of CE with TATA-boxes are revealed if the in result of the functional overlap between two components of CE overlap of sequences leads to the emergent appearance of a new function. In the function any mutations in any of the components of CE can not be compensated by any substitutions in the other component of CE. Thus, the HS can stabilize not only the minimization of the functional overlapping of traits (the classical HS of Vavilov and Sobolev) [1], but also the emergence of a new function as a by-effect of functional overlap. Otherwise, the HS may be lost by triggering the autoadaptive mechanism of the compensatory divergence [1].

Conclusion: The work of the Meyen school showed [2] but not explained that clear HS are well identified on the molecular-biological (suborganismal) and ecological (or supraorganismal) levels, that is, where both mechanisms of stabilization of the HS mast work best. Complex behavior and stress that appear on the organismal level open up wide opportunities for both compensation and overlapping of functions, which makes the preserve of the HS in evolution on organismal level is problematic.

Acknowledgements: Supported by the grant 0324-2018-0017 and IP 0324-2018-0021.

\section{References}

1. Suslov V.V. et al. (2016) Vavilov's homologous series as evolutionary force. BGRS/SB'2016:307.

2. Chebanov S.V. (2017) Meronomy of S.V. Meyen: to the 40th anniversary of formulation. Lethaea rossica. 14:64-92. 